\title{
Characterization of Naturally-Occurring Humoral Immunity to AAV in Sheep
}

\author{
Joseph Tellez' ${ }^{1}$ Kim Van Vliet ${ }^{2}$, Yu-Shan Tseng ${ }^{2}$, Jonathan D. Finn ${ }^{3}$, Nick Tschernia ${ }^{1}$, Graça Almeida- \\ Porada $^{4}$, Valder R. Arruda ${ }^{3}$, Mavis Agbandje-McKenna ${ }^{2}$, Christopher D. Porada ${ }^{4}$
}

1 Department of Animal Biotechnology, University of Nevada, Reno, Nevada, United States of America, 2 Department of Biochemistry and Molecular Biology, University of Florida, Gainesville, Florida, United States of America, 3 University of Pennsylvania School of Medicine, the Children's Hospital of Philadelphia, Philadelphia, Pennsylvania, United States of America, 4 Wake Forest Institute for Regenerative Medicine, Winston-Salem, North Carolina, United States of America

\begin{abstract}
AAV vectors have shown great promise for clinical gene therapy (GT), but pre-existing human immunity against the AAV capsid often limits transduction. Thus, testing promising AAV-based GT approaches in an animal model with similar pre-existing immunity could better predict clinical outcome. Sheep have long been used for basic biological and preclinical studies. Moreover, we have re-established a line of sheep with severe hemophilia A (HA). Given the impetus to use AAV-based GT to treat hemophilia, we characterized the pre-existing ovine humoral immunity to AAV. ELISA revealed naturally-occurring antibodies to AAV1, AAV2, AAV5, AAV6, AAV8, and AAV9. For AAV2, AAV8, and AAV9 these inhibit transduction in a luciferase-based neutralization assay. Epitope mapping identified peptides that were common to the capsids of all AAV serotypes tested (AAV2, AAV5, AAV8 and AAV9), with each animal harboring antibodies to unique and common capsid epitopes. Mapping using X-ray crystallographic AAV capsid structures demonstrated that these antibodies recognized both surface epitopes and epitopes located within regions of the capsid that are internal or buried in the capsid structure. These results suggest that sheep harbor endogenous AAV, which induces immunity to both intact capsid and to capsid epitopes presented following proteolysis during the course of infection. In conclusion, their clinically relevant physiology and the presence of naturally-occurring antibodies to multiple AAV serotypes collectively make sheep a unique model in which to study GT for HA, and other diseases, and develop strategies to circumvent the clinically important barrier of pre-existing AAV immunity.
\end{abstract}

Citation: Tellez J, Van Vliet K, Tseng Y-S, Finn JD, Tschernia N, et al. (2013) Characterization of Naturally-Occurring Humoral Immunity to AAV in Sheep. PLoS ONE 8(9): e75142. doi:10.1371/journal.pone.0075142

Editor: Jianming Qiu, University of Kansas Medical Center, United States of America

Received May 15, 2013; Accepted August 9, 2013; Published September 24, 2013

Copyright: $\odot 2013$ Tellez et al. This is an open-access article distributed under the terms of the Creative Commons Attribution License, which permits unrestricted use, distribution, and reproduction in any medium, provided the original author and source are credited.

Funding: This work was supported by NIH-HD43038 and Wake Forest University Health Sciences Translational Science Institute 20667 Clinical Pilot Award to CDP; NIH- PO1HL64190 to VRA; NIH R01 GM082946 and University of Florida College of Medicine Research Funds to MA-M. The funders had no role in study design, data collection and analysis, decision to publish, or preparation of the manuscript.

Competing interests: The authors have declared that no competing interests exist.

*E-mail: cporada@wakehealth.edu

\section{Introduction}

Adeno-associated viruses (AAVs) have attracted considerable interest in the field of gene therapy because they possess many characteristics that make them excellent vectors for gene transfer. Their genome is easily manipulated, AAV particles can be purified at very high titers, and they can then be lyophilized for easy handling/storage [1-3]. AAV efficiently transduces both proliferating and quiescent cells, and numerous serotypes exist in nature with differing tropisms, permitting some degree of tissue-targeting [4-7]. Also, the general consensus has been that AAVs may be inherently safer than many other viral-based vectors, since they are nonpathogenic and possess relatively low innate immunogenicity. Because genomic integration of AAV vectors is rare [8-11], the risk of insertional mutagenesis with AAV vectors is greatly reduced compared to retroviruses. These collective features have enabled AAV vectors to effectively correct a wide range of diseases in animal models, which, in turn, has prompted numerous clinical trials, in the hopes of safely achieving longterm expression of a variety of therapeutic proteins in human patients. An ongoing clinical gene therapy trial for hemophilia $B$ $[12,13]$ clearly highlights the tremendous potential of $A A V-$ based vectors for the treatment of human disease.

However, many of the serotypes of AAV commonly employed in gene therapy procedures ubiquitously infect humans, generating pre-existing immunity against the AAV capsid proteins that precludes efficient transduction following intravascular administration, and/or induces CTL responses to the transduced target tissue [14-21]. While newborn dogs have 
been reported to exhibit pronounced selective immunity to AAV6 [22], most animals, with the exception of some nonhuman primates [19,23] and, perhaps the pig [24], do not appear to harbor very robust pre-existing immunity/ endogenous antibodies to many of the serotypes of AAV commonly employed as gene delivery vectors. This lack of preexisting immunity to AAV could explain, in part, why many highly successful studies conducted in a variety of animal models have not translated into clinical success when similar approaches have been applied to human patients $[25,26]$. Sheep have been used for decades as a model to study a broad range of disease states, and a high degree of clinical predictability has consistently been observed. Recently, sheep were utilized as a large animal model for myocardial gene delivery using molecular cardiac surgery with recirculating delivery (MCARD) [27], as well as for testing rAAV2/1SERCA2a vectors, in an experimental heart failure model [28]. Novel in utero gene therapy studies aimed at correcting congenital diseases that develop perinatally recently also utilized sheep as the large animal model [29]. Moreover, we have re-established a line of sheep with severe hemophilia $A$ with a null mutation in the FVIII gene [30] to provide a suitable large animal model for testing novel rAAV vectors for this condition. A recent publication provided evidence that commercially available sheep sera may harbor very low levels of antibodies to a limited number of AAV serotypes [24], suggesting at least a subset of sheep may harbor immunity to AAV. Here we report studies performed to address whether sheep harbor naturally-occurring antibodies to the same serotypes of AAV as healthy humans [20,21], and to characterize the nature of this pre-existing immunity. Enzymelinked immunosorbent assays (ELISA) demonstrated the existence of naturally occurring antibodies against several AAV serotypes, AAV1, AAV2, AAV8, and AAV9. In addition, sera from three of the sheep screened neutralized transduction by AAV2, AAV8, and AAV9 to different levels, with AAV2 being the most affected. These observations recapitulate reports for the human response against the AAVs, with antibodies against AAV2 being the most prevalent in the human population [20]. Finally, peptide array based epitope mapping identified common AAV serotype antigenic epitopes that were recognized by different sheep sera as well as those that were unique to each sheep. Similar to the human immune response against the $A A V s$, the sheep reactivity against the different AAV serotypes was varied. To our knowledge, this is the first study to delineate, in a large animal (sheep) model, the epitopes within the AAV capsid that are responsible for triggering naturally-occurring functional antibodies to multiple serotypes of AAV commonly employed as gene therapy vectors. The close parallels between human and sheep physiology and the presence of these antibodies, suggest that sheep may represent an ideal large animal model in which to study gene therapy in the context of pre-existing immunity to $\mathrm{AAV}$, and to develop novel strategies for circumventing this clinically important immunologic barrier.

\section{Materials and Methods}

\section{Ethics Statement}

This study was approved by the University of Nevada, Reno Institutional Animal Care and Use Committee.

\section{Indirect enzyme-linked immunosorbent assay (ELISA) detection of anti-AAV-antibodies in sheep serum}

Serum collected from the peripheral blood of six healthy adult female Merino-Rambouillet sheep bred and raised at the University of Nevada Agricultural Experiment Station was used as the primary antibody in an indirect ELISA. Sheep IgG (Rockland, Gilbertsville, PA, USA) was used to prepare a standard curve (31.25-4000ng/ml) and AAV1-FIX, AAV2-FIX, AAV5-GFP, AAV6-GFP, AAV8-Ova and AAV9-Ova (AAV5 and 6 were purchased from Vector Biolabs, Philadelphia, PA, USA; AAV1, 2, 8, and 9 were kindly provided by Dr. Roland Herzog) served as the antigen $\left(2.5 \times 10^{9}\right.$ vector genomes/well). Both the antibody and virus samples were fixed to the appropriate wells of a 96-well plate with $50 \mu \mathrm{l}$ Coating Solution (KPL, Gaithersburg, MD, USA), the plate was sealed, and then incubated overnight at $4^{\circ} \mathrm{C}$. The plate was washed 3 times with phosphate-buffered saline (PBS: $137 \mathrm{mM} \mathrm{NaCl}, 2.7 \mathrm{mM} \mathrm{KCl}$, $1.5 \mathrm{mM} \mathrm{KH}_{2} \mathrm{PO}_{4}, 8 \mathrm{mM} \mathrm{NaH}_{2} \mathrm{PO}_{4}$ ) containing $0.05 \%$ Tween-20, and blocked with blocking/dilution buffer (PBS, 6\% BSA, 0.05\% Tween-20) for $1 \mathrm{hr}$ at RT. The plate was again washed three times with PBS containing $0.05 \%$ Tween-20. Sheep serum was diluted 1:20 in blocking/dilution buffer, and $50 \mu \mathrm{l}$ was added to each of the experimental wells. Wells containing no viral particles served as controls for non-specific binding of sheep serum and secondary antibody to the microplate wells. The plate was incubated for $2 \mathrm{hr}$ at $37^{\circ} \mathrm{C}$ and then washed three times in PBS containing $0.05 \%$ Tween-20. Rabbit-anti-sheep IgG conjugated to horse radish peroxidase (HRP) secondary antibody (Southern Biotech, Birmingham, AL, USA) was diluted 1:500 in blocking/dilution buffer and added to the wells to detect bound antibodies in the sheep sera. The plate was incubated again for $2 \mathrm{hr}$ at $37^{\circ} \mathrm{C}$ and washed three times in PBS containing $0.05 \%$ Tween-20. One hundred microliters of SIGMAFAST TM OPD substrate (ophenylenediaminedihydrochloride, Sigma-Aldrich, St. Louis, $\mathrm{MO}$, USA) was added to each well, and the plate was incubated for $1 \mathrm{hr}$ at $37^{\circ} \mathrm{C}$, with OD readings taken every 15 minutes in a 3550-UV Microplate Reader (Bio-Rad, Hercules, CA, USA) until the standard curve was linear in a semi-log scale.

\section{Luminescence-based AAV neutralization assay}

HEK-293 cells were seeded at 20,000 cells/well in $200 \mu \mathrm{l}$ of Dulbecco's Modified Eagle Medium (DMEM; Invitrogen/Life Technologies, Grand Island, NY, USA) containing 10\% fetal bovine serum (FBS; HyClone/Thermo Scientific, Logan, UT, USA), 1\% glutamine (Invitrogen/Life Technologies), and 1\% penicillin/streptomycin (Invitrogen/Life Technologies) in 96-well plates (E\&K Scientific Products, Inc., Santa Clara, CA, USA). Cells were then incubated overnight at $37^{\circ} \mathrm{C}, 5 \% \mathrm{CO}_{2}$.

On the following day, sera from the 3 sheep exhibiting different titers of antibodies (lowest, medium, and highest) to 
the various AAV serotypes were thawed on ice and then heatinactivated for 30 minutes at $56^{\circ} \mathrm{C}$. A series of dilutions (from $1: 3.1$ to $1: 3160$ ) of eah serum sample was then prepared in heat-inactivated FBS, with pooled normal human plasma diluted in the same fashion used as a positive control/standard. AAV2, AAV8, and AAV9 vectors encoding the Renilla luciferase gene under the control of the cytomegalovirus/ chicken beta-actin hybrid (CBA) promoter were used. Eighteen microliters of each diluted AAV vector (8e7 vg for AAV2, 1.5e9 $\mathrm{vg}$ for AAV 8 and 9 ) was mixed with $18 \mu$ l of each of the diluted serum samples, and incubated for $1 \mathrm{hr}$ at $37^{\circ} \mathrm{C}$. An aliquot $(7.5 \mu \mathrm{l})$ of each of the serum/AAV vector mixtures was then transferred to an individual well of the tissue culture plate containing the HEK-293 cells, plating each sample dilution in triplicate (AAV2 $\mathrm{MOI}=\sim 800, \mathrm{AAV} 8 / 9 \mathrm{MOI}=\sim 15,000)$. The plate was incubated overnight $(16-24 \mathrm{hr})$ at $37^{\circ} \mathrm{C}, 5 \% \mathrm{CO}_{2}$.

The next morning, 5x Renilla luciferase lysis buffer (Promega Corp., Madison, WI, USA) was thawed, diluted to a $1 \mathrm{x}$ working solution with $\operatorname{diH}{ }_{2} \mathrm{O}$, and kept on ice until use. The medium was removed from the HEK-293 cell plates, and each well was gently washed with $200 \mu$ l of $1 x$ PBS. Forty microliters of $1 x$ lysis buffer was added to each well, and the plates were then agitated for 15 minutes at room temperature on a Clay Adams Nutator Mixer (BD Diagnostics, Franklin Lakes, NJ, USA). The Renilla luciferase assay buffer was thawed and used to prepare a $1 \mathrm{x}$ solution of Renilla luciferase assay substrate (both from Promega). Following priming of the luminometer (GloMax®-96 Microplate Luminometer, Promega Corp.) with substrate buffer, the plates were inserted and read with an integration time of 2 seconds, an injection volume of $50 \mu \mathrm{l}$, an injection rate of $333 \mu \mathrm{l} / \mathrm{second}$, and a delay between injection and measurement of 1 second. The data generated was automatically saved within a Microsoft Excel spreadsheet.

\section{Peptide library generation and peptide epitope- mapping}

ProArray ${ }^{\mathrm{TM}}$ peptide microarrays containing 557 15mer peptides overlapping by 10 amino acids and covering the entire VP1 sequence from the capsids of AAV2, AAV5, AAV8, and AAV9 (Prolmmune, Inc., Oxford, UK) were used to screen reactivity of individual sheep sera. All ProArrays ${ }^{\mathrm{TM}}$ were blocked for $2 \mathrm{hr}$ using validated, proprietary blocking buffer, and were then incubated with the individual sheep sera (collected from the 3 sheep used for the Luminescence-Based AAV neutralization assay detailed above) diluted in blocking buffer (1:200 dilution, total assay volume $400 \mu \mathrm{L}$ ), with a fluorescently labeled secondary antibody, which binds selectively to an antigen-specific antibody (i.e. anti-sheep) (control sample) or with blocking buffer only (control buffer). The ProArrays ${ }^{\mathrm{TM}}$ were washed five times with blocking buffer and five times with PBS, followed by incubation with Cy3-labeled AffiniPure Rabbit AntiSheep-lgG $(\mathrm{H}+\mathrm{L})$ secondary antibody (Stratech Scientific, Ltd., Newmarket Suffolk, England) at a concentration of $1 \mu \mathrm{g} / \mathrm{ml}$. Following incubation with the secondary antibody, five washing steps with blocking buffer were performed, followed by five washing steps with PBS, and five washing steps with analytical grade water. The ProArrays ${ }^{\mathrm{TM}}$ were then thoroughly dried and scanned using a high-resolution fluorescence scanner
(Innoscan 700; Innopsys, Carbonne, France). Laser settings and applied resolution were identical for all measurements performed. The resulting images were processed and analyzed using the integrated Mapix spot-recognition software (Arrayit Corporation (ARYC), Sunnyvale, CA), showing the signal intensity (range between 0 and 65536 Light Units) as single measurements for each peptide. Each spot-feature was analyzed for signal intensities for pixels within recognized spots and for the pixels surrounding the recognized spots (background). The median of signal intensities of the background was subtracted from the median of signal intensities for pixels within recognized spots, resulting in corrected median values (signal minus background). Mean values of the corrected median of signal intensities from 3 identical subarrays were used for the data analysis. The signals from the control sample (anti-sheep secondary antibody) were subtracted from the sheep sera signals and peptides with intensity values greater than 7,500 LU were considered positive binders. The VP and capsid regions of the peptides identified by the peptide microarray screening were visualized in the structures of AAV2, AAV5, AAV8, and AAV9 capsids, determined by X-ray crystallography (PDB identifiers 1LP3, 3NTT, 2QA0, and 3UX1, respectively), using the Coot program [31] and images to show their locations were generated using the PyMol program (The PyMOL Molecular Graphics System, Version 1.3, Schrödinger, LLC.).

\section{Results}

\section{Detection of naturally-occurring antibodies to AAV in sheep}

To assess whether sheep harbor naturally-occurring antibodies against the serotypes of AAV commonly employed as gene therapy vectors, ELISAs were performed on sera from a panel of 6 healthy Merino-Rambouillet sheep using AAV1, AAV2, AAV5, AAV6, AAV8, and AAV9 particles as the antigen. As shown in Figure 1, these analyses revealed that healthy sheep harbored naturally-occurring antibodies to all 6 AAV serotypes tested. The titers against the different serotypes varied greatly from sheep-to-sheep, similar to the observation of human sera reactivities to AAVs. Specifically, one sheep (Sheep F) exhibited a very high level $(>5900 \mathrm{ng} / \mathrm{ml})$ of $\mathrm{lgG}$ against all 6 AAV serotypes tested. In contrast, levels of antiAAV IgG in the remaining 5 sheep were varied, with Sheep C exhibiting moderate/low (992-1530ng/ml) lgG levels against all 6 AAV serotypes, Sheep D moderate/low $(500-1637 \mathrm{ng} / \mathrm{ml}) \mathrm{lgG}$ levels against all serotypes except AAV9, Sheep B and $E$ moderate/low (335-1972ng/ml) IgG levels against only 3-4 of the tested serotypes, and sheep A only low $(\leq 500 \mathrm{ng} / \mathrm{ml}) \mathrm{lgG}$ levels against all 6 AAV serotypes.

\section{Anti-AAV antibodies in sheep neutralize transduction}

To assess the functional significance of the anti-AAV antibodies in the sheep sera in the context of AAV-based gene delivery, a luciferase-based neutralization assay was performed (see Materials and Methods) on sera from three sheep exhibiting, low (sheep A), medium (sheep C), and high (sheep F) IgG titers against AAV2, AAV8, and AAV9. As shown 


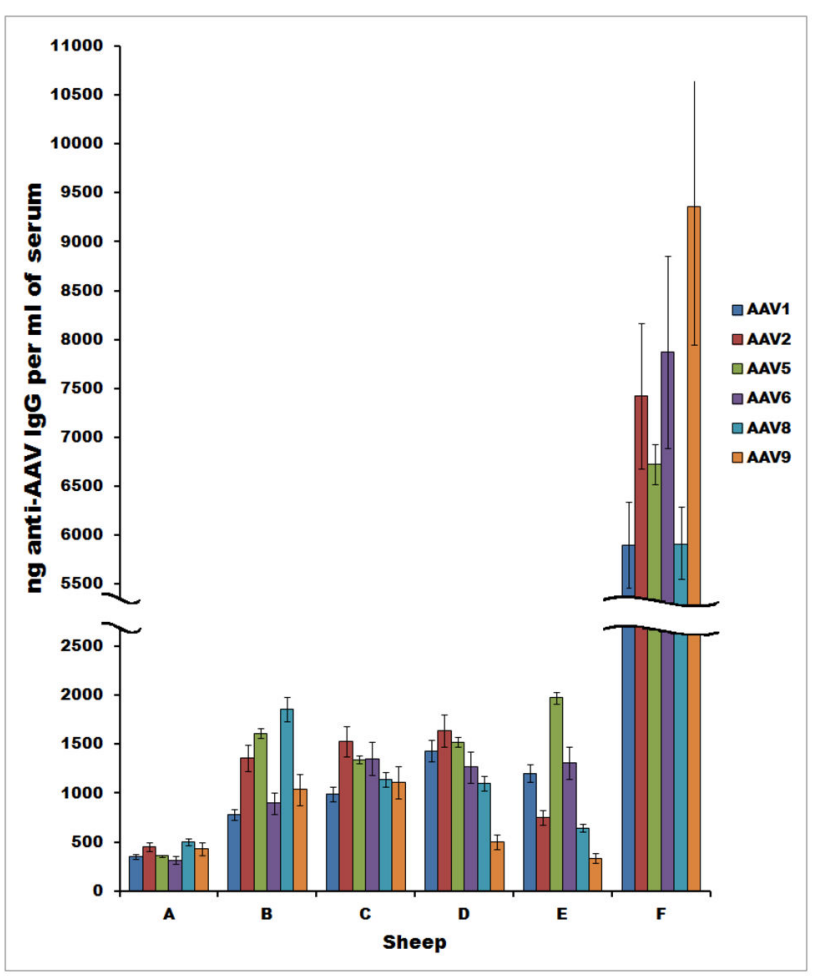

Figure 1. Presence in sheep of antibodies that recognize common AAV serotypes. ELISAs were performed on sera from a panel of 6 healthy sheep using AAV1, AAV2, AAV5, AAV6, AAV8, or AAV9 particles as the antigen. Results are presented as concentration of IgG against each AAV serotype in each individual animal's serum. All samples were run in triplicate, and the depicted values represent the mean of 3 separate experiments.

doi: 10.1371/journal.pone.0075142.g001

in Figure 2, despite having widely disparate levels of $\lg G$ against AAV2 by ELISA, sera from all three of the animals tested were neutralizing (1:100 to $1: 316$ dilution) against AAV2, similar to the high neutralizing activity seen with pooled human plasma against this AAV serotype. In agreement with the IgG levels seen by ELISA (Figure 1), only the sera from Sheep $F$ was neutralizing against AAV8 and AAV9 (1:31, and 1:100 dilution, respectively), while the sera from Sheep $A$ and Sheep C were not neutralizing (1:1 to1:3 dilution) against these two AAV serotypes. While the pooled human plasma neutralized both AAV8 and AAV9, the neutralizing titers against these two serotypes were lower than those observed against AAV2.

\section{Identification of capsid epitopes recognized by anti- AAV IgG in sheep}

Our results demonstrated the presence of antibodies recognizing multiple AAV serotypes, raising the question of whether this seemingly broad immunity to AAV was the result of antibodies against an epitope(s) common to all of the tested AAV serotypes, or whether sheep harbor antibodies that recognize multiple distinct epitopes that are unique to each serotype's capsid. To address this question, we performed epitope mapping to define the peptides within the capsids of AAV2, AAV5, AAV8, and AAV9 recognized by these antibodies. As detailed in the Materials and Methods, a peptide library covering the entire VP1 sequence from the capsids of AAV2, AAV5, AAV8, and AAV9, was synthesized by Prolmmune, Inc. (Oxford, UK) and probed with sera from the three sheep utilized for the neutralization assay (sheep $A$, sheep $C$, and sheep F). The screening resulted in the identification of numerous immunogenic epitopes within the capsids of these viruses as positive hits. As can be seen in Figure 3 , the antibodies present within the serum from each sheep recognized multiple distinct epitopes within the capsid, with varying degrees of affinity, the majority of which were common amongst the three sheep tested. The sequence information for each of the most immunogenic peptide epitopes identified with these arrays are shown in Table 1, along with the serotypes of AAV containing the given epitope, and the epitope's amino acid position within the respective VP1. Regions of the capsid most likely to contain antigenic regions are included in peptides $26,44,45,323,326,327,354,401$, $402,450,451$, and 485. Although many of the recognized epitopes in Table 1 were common to the capsids of all AAV serotypes tested, each animal also harbored antibodies to epitopes that were unique to each specific capsid, with Sheep $F$ having the most reactivity, consistent with the ELISA and neutralizing antibody data. Using the AAV capsid structures determined by X-ray crystallography for AAV2, AAV5, AAV8, and AAV9 [32-34] the positive hit peptides (Table 1) were mapped on the capsid VP to determine their structural locations (Table 1 and Figure 4). Surprisingly, most of the epitopes were located within regions of the VP that are internal or buried within the capsid in conserved secondary structure elements (Figures 4B, 4D, 4F and 4H). However, consistent with the recognition of the AAV capsids in the ELISA and neutralization assay results, a number of the peptides recognized were located on the capsid surface and in regions analogous to previously mapped neutralizing antigenic epitopes in AAV2 and in AAV8 [35-37] (Table 1, Figures 4A, $4 \mathrm{C}, 4 \mathrm{E}$ and $4 \mathrm{G}$ ). These results suggest that sheep harbor endogenous AAV, which induces immunity to both intact capsids and capsid epitopes that are presented following proteolysis during the course of infection.

\section{Discussion}

\section{Sheep as an animal model for studying the immune response to AAV gene therapy vectors}

Preclinical studies in both small and large animal models have demonstrated that AAV-mediated gene transfer is safe and has resulted in long-term gene expression and phenotypic correction of a wide range of disorders (e.g. [2,8,9,11,12,27-29,38-40]). However, the host immune response represents a formidable barrier to achieving therapeutic efficacy in the clinic, due largely to the presence of pre-existing immunity to AAV capsids in humans as a result of previous exposure to AAV. A recent publication [24] provided evidence that sera from a variety of animals commonly 


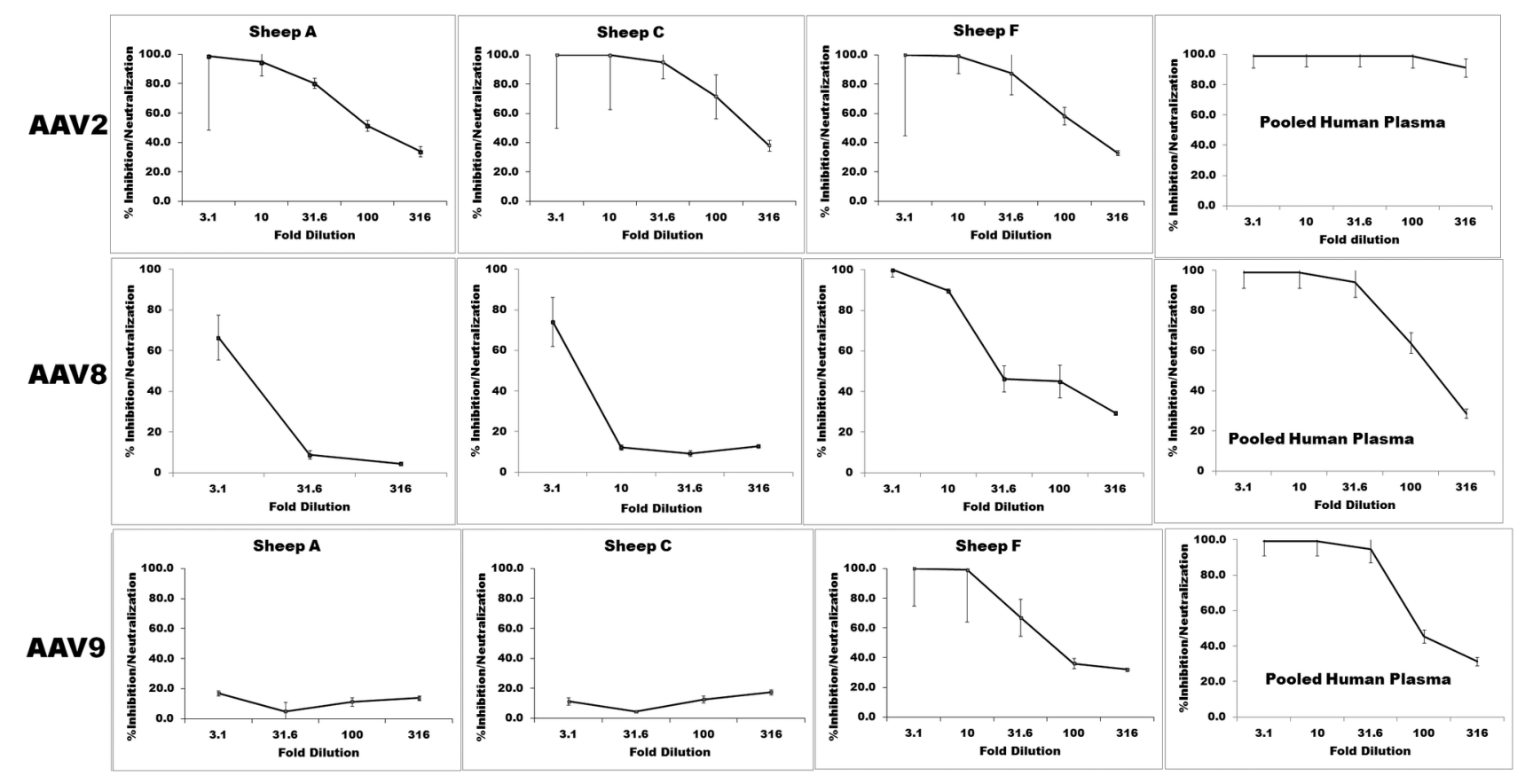

Figure 2. Neutralizing activity of anti-AAV antibodies. To assess the functional significance of the detected antibodies in the context of AAV-based gene delivery, a luciferase-based neutralization assay was performed on sera from sheep A, sheep C, and sheep F against AAV2, AAV8, and AAV9. Pooled normal human plasma diluted in the same fashion served as a positive control/ standard. Data are presented as the titer (dilution) of the serum from each sheep versus the percent inhibition observed when a known quantity of luciferase-encoding AAV vector of each serotype was exposed to serum from each sheep and then used to transduce HEK-293 cells in vitro. The values presented are the mean obtained running all samples in triplicate \pm SEM.

doi: 10.1371/journal.pone.0075142.g002

employed for biomedical research, including sheep, harbor neutralizing antibodies to some serotypes of AAV. This study thus identified animal species suited for studying the immunologic aspects of AAV-based gene delivery evaluating strategies to circumvent pre-existing AAV immunity. Sheep have long been used as a model to study normal development and physiology, as well as a broad range of disease states, and results obtained with this model have consistently yielded a high degree of clinical predictability in humans. In this study, the humoral immunity to AAV observed in normal healthy sheep, based on capsid ELISA, mimicked the marked individual-to-individual titer variability of antibodies against AAV capsid that has been observed in the human population [20]. The neutralization assay from the three selected sheep with variable IgG response further confirmed the similarity between the sheep and human pre-existing AAV capsid immunity response when tested against AAV2, AAV8, and AAV9. These analyses revealed that, like most humans, sheep harbor relatively high titer neutralizing antibodies (NAB) to AAV2, but varied titers against AAV8 and AAV9. Prior studies have shown that even low levels of neutralizing antibody (1:5 to $1: 10)$ were sufficient to completely abrogate AAV-mediated transduction $[38,41]$, and even non-neutralizing antibodies negatively impacted upon transduction in vivo [42], consistent with our observations. These observations establish sheep as the first experimental animal model with significant $N A B$ and $\lg G$ to
AAV2 and AAV8, and thus support the use of sheep for future studies of the immune response to AAV gene therapy vectors.

\section{Specificity and cross-reactivity is common to sheep and AAV serotypes}

Peptide-based array epitope screening defined peptides within the capsids of AAV2, AAV5, AAV8, and AAV9 recognized by the three sheep sera tested. The reactivity profiles were very similar between the sheep (Figure 3) with sheep $F$, containing the highest AAV antibody titer against the different serotypes, showing strongest reactivity against all the serotypes. Sheep F harbored antibodies that recognized multiple distinct epitopes that are unique to each capsid serotype as well as those that were common to all of the tested AAV serotypes (Table 1). The low and medium AAV antibody titer sera (Sheep A and C, respectively) reacted only to the same peptides as sheep $F$. The observed commonality in peptide epitopes implies that the amino acid sequences recognized, which are conserved among the human and nonhuman primate $\mathrm{AAVs}$ tested, are also conserved in the endogenous ovine AAVs. The data also suggests that these sheep have been exposed to the same or similar viruses.

Several epitopes that were recognized by peptide mapping are located in the unique N-terminus of VP1 and VP1/VP2 overlap which are not ordered in crystal structures determined for the AAV capsids and are also proposed to be buried inside 


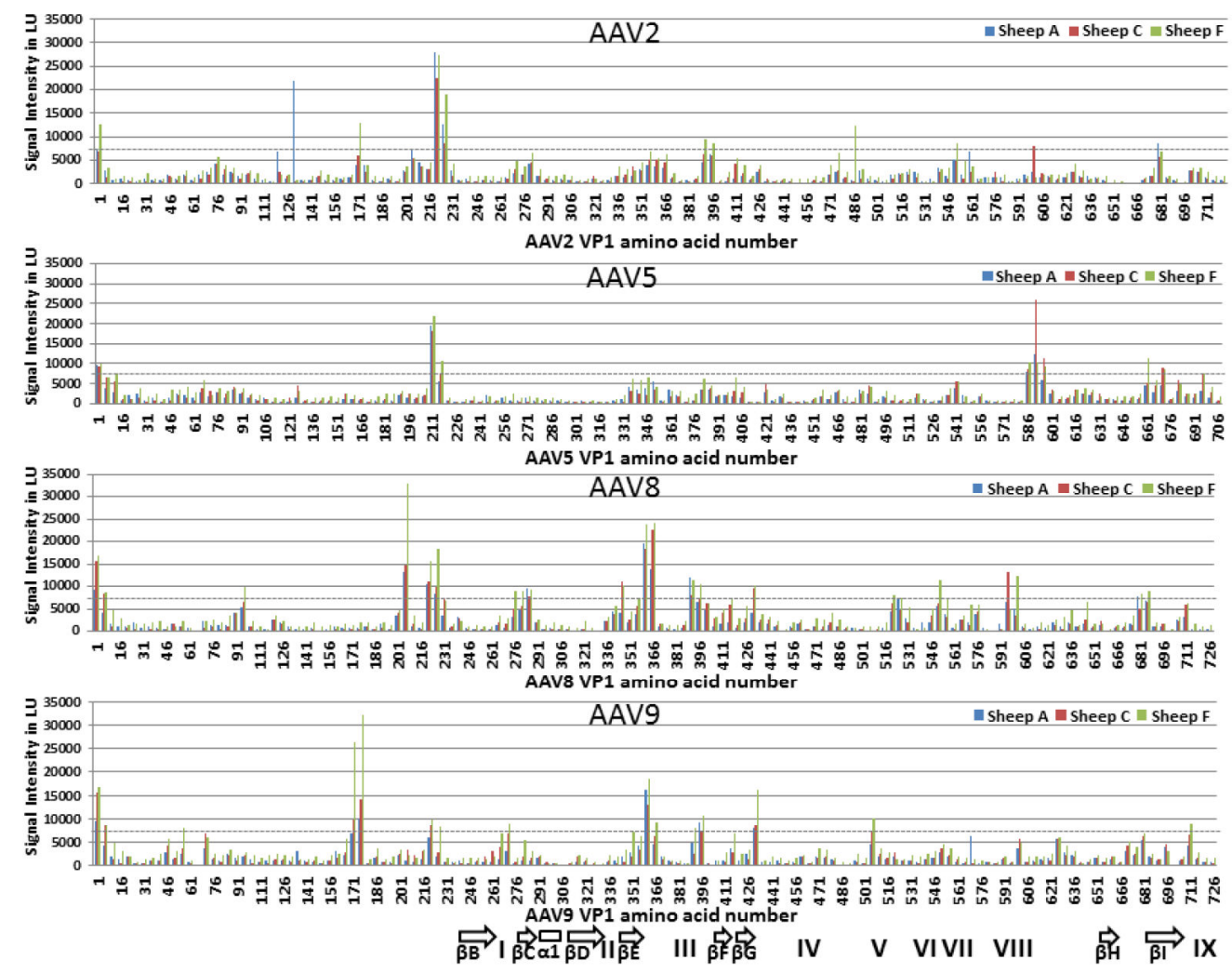

\section{Figure 3}

Figure 3. Identification of peptides responsible for antibody binding to AAV. To identify the epitopes within the AAV capsid recognized by the antibodies present within the sera of healthy sheep, a comprehensive peptide library covering the entire VP1 sequence from the capsids of AAV2, AAV5, AAV8, and AAV9 was probed with sera from the three sheep utilized for the neutralization assay (sheep A, sheep $C$, and sheep F). The histograms depicted in this figure show the results obtained when arrays containing peptides for AAV2, AAV5, AAV8, or AAV9 were probed with sera from sheep A, sheep C, or sheep F. The histogram was plotted based on the amino acid number of the first amino acid in the reactive peptide. VP1 numbering for each serotype was used. doi: 10.1371/journal.pone.0075142.g003

the capsid. In addition, a peptide mapping study for AAV2 by Moskalenko, et al, identified epitopes that were buried in the AAV2 capsid structure, as well as surface associated epitopes [43]. Some of the epitopes identified by Moskalenko, et al, were also identified in this study (Table 1). The AAV capsid in solution is dynamic and undergoes conformational changes upon binding to cellular receptors and uncoating. These changes during the virus life cycle may expose antigenic sites that were previously hidden within the capsid. Zadori, et al [44] have shown that the unique region of VP1 (VP1u), which is internal in the capsid, becomes externalized during the course of infection, possesses phospholipase activity, and is required for infectivity. Recently, specific protein sequence motifs were identified in VP1u that play a role in intracellular trafficking and nuclear localization [45]. Peptides most likely to be antigenic in this region include peptides 26, 323, 450, and 451 (shown in Figure 3). Peptide 26 is an AAV2 peptide that includes the $\mathrm{N}$ - terminus of the $\mathrm{VP}_{1 / 2}$ common region. Peptides 450 and 451 are AAV9 peptides that are similar to peptide 34 which is an AAV2 peptide that has previously been shown to contain the epitope for A69 antibody [37]. Peptide 323 (shown in Figure 3) is an AAV8 peptide that includes part of VP2, as well as the Nterminus of VP3. These regions of the capsid are not detectable in the available structures of AAV serotypes solved by X-ray crystallography (Table 1 , shaded grey). A similar study using pig sera to evaluate antigenic regions on the capsid of Swine Vesicular Disease Virus (SVDV) also identified novel antigenic regions that were internal or located at subunit interfaces [46]. Common epitopes were recognized for conserved regions of AAV2, AAV5, AAV8, and AAV9 (Table 1, epitope $a, b, c, d$ and e). This cross-reactivity is consistent with reports where immunization with AAV2 capsids led to formation of antibodies that also recognized AAV8 capsids and interfered with subsequent AAV8-mediated gene transfer [47], and with a 
Table 1. Positive Binders.

\begin{tabular}{|c|c|c|c|c|c|c|}
\hline \multirow{2}{*}{$\begin{array}{l}\text { Sheep } \\
F\end{array}$} & \multirow{2}{*}{$\begin{array}{l}\text { ID } \\
1\end{array}$} & \multicolumn{2}{|c|}{ Epitope Sequence } & \multirow{2}{*}{$\begin{array}{l}\text { AAV Serotype } \\
\text { AAV2 }\end{array}$} & \multirow{2}{*}{$\begin{array}{l}\text { Amino acid\# (VP1 \#'s) } \\
1-15\end{array}$} & \multirow[t]{2}{*}{ Epitope/Location } \\
\hline & & $\mathbf{a}$ & MAADGYLPDWLEDTL & & & \\
\hline$A, C, F$ & 145 & & MSFVDHPPDWLEEVG & AAV5 & $1-15$ & \\
\hline$A, C, F$ & 287 & a & MAADGYLPDWLEDNL & AAV8, AAV9 & $1-15,1-15$ & \\
\hline $\mathrm{C}, \mathrm{F}$ & 288 & & YLPDWLEDNLSEGIR & AAV8 & $6-20$ & \\
\hline $\mathrm{F}$ & 304 & & ADAEFQERLQEDTSF & AAV8 & $96-110$ & \\
\hline A & 26 & & LVEEPVKTAPGKKRP & AAV2 & $131-145$ & \\
\hline $\mathrm{F}$ & 34 & & LNFGQTGDADSVPDP & AAV2 & $171-185$ & A69/ND \\
\hline $\mathrm{C}, \mathrm{F}$ & 450 & & LNFGQTGDTESVPDP & AAV9 & $207-225$ & \\
\hline$A, C, F$ & 451 & & TGDTESVPDPQPIGE & AAV9 & $208-225$ & \\
\hline A & 41 & & GSGAPMADNNEGADG & AAV2 & $209-225$ & \\
\hline$A, C, F$ & 323 & & AGGGAPMADNNEGAD & AAV8 & $210-225$ & \\
\hline$A, C, F$ & 187 & b & VGNASGDWHCDSTWM & AAV5 & $211-225$ & IP-IS \\
\hline $\mathrm{F}$ & 188 & b & GDWHCDSTWMGDRVV & AAV5 & $216-230$ & IP-IS \\
\hline$A, C, F$ & 44 & b & VGNSSGNWHCDSTWM & AAV2 & $221-235$ & IP-IS \\
\hline A, C, F & 326 & b & GVGSSSGNWHCDSTW & AAV8 & $221-235$ & IP-IS \\
\hline $\mathrm{F}$ & 460 & b & VGSSSGNWHCDSQWL & AAV9 & $221-235$ & IP-IS \\
\hline$A, C, F$ & 45 & b & GNWHCDSTWMGDRVI & AAV2 & $226-240$ & IP-IS \\
\hline$A, C, F$ & 327 & b & SGNWHCDSTWLGDRV & AAV8 & $226-240$ & IP-IS \\
\hline $\mathrm{F}$ & 461 & b & GNWHCDSQWLGDRVI & AAV9 & $226-240$ & IP-IS \\
\hline $\mathrm{F}$ & 467 & & DNAYFGYSTPWGYFD & AAV9 & $271-285$ & B \\
\hline $\mathrm{F}$ & 337 & & FGYSTPWGYFDFNRF & AAV2, AAV5, AAV8, AAV9 & $273-287,264-278,276-290,275-289$ & A20-1/B \\
\hline $\mathrm{F}$ & 338 & & PWGYFDFNRFHCHFS & AAV2, AAV8, AAV9 & $278-292,281-295,280-294$ & B \\
\hline$A, C, F$ & 339 & & DFNRFHCHFSPRDWQ & AAV2, AAV8, AAV9 & $283-297,286-300,285-299$ & B \\
\hline $\mathrm{C}, \mathrm{F}$ & 351 & & FTDSEYQLPYVLGSA & AAV2, AAV8 & $343-357,346-360$ & $\mathrm{MK} / \mathrm{IS} / \mathrm{B}$ \\
\hline$A, C, F$ & 354 & c & HQGCLPPFPADVFMI & AAV8 & $361-375$ & B \\
\hline$A, C, F$ & 485 & c & EGCLPPFPADVFMIP & AAV9 & $361-375$ & B \\
\hline$A, C, F$ & 355 & c & PPFPADVFMIPQYGY & AAV2, AAV8, AAV9 & $363-377,366-380,365-379$ & A20-2/B \\
\hline $\mathrm{F}$ & 486 & c & PFPADVFMIPQYGYL & AAV2, AAV8, AAV9 & $364-378,367-381,366-380$ & A20-2/B \\
\hline$A, C, F$ & 360 & d & GRSSFYCLEYFPSQM & AAV2, AAV8, AAV9 & $388-402,391-405,390-404$ & IP-IS \\
\hline $\mathrm{F}$ & 491 & d & RSSFYCLEYFPSQML & AAV9 & $391-405$ & IP-IS \\
\hline $\mathrm{F}$ & 78 & d & SFYCLEYFPSQMLRT & AAV2, AAV8, AAV9 & $391-405,394-408,393-407$ & MK/IS \\
\hline $\mathrm{F}$ & 361 & d & YCLEYFPSQMLRTGN & AAV2, AAV8, AAV9 & $393-407,396-410,395-409$ & $\mathrm{MK} / \mathrm{IS}$ \\
\hline$A, F$ & 492 & d & CLEYFPSQMLRTGNN & AAV2, AAV8, AAV9 & $394-408,397-411,396-410$ & MK/IS \\
\hline $\mathrm{F}$ & 79 & & EYFPSQMLRTGNNFT & AAV2 & $396-410$ & MK/IS \\
\hline $\mathrm{F}$ & 365 & & YTFEDVPFHSSYAHS & AAV8 & $416-430$ & IS \\
\hline $\mathrm{C}, \mathrm{F}$ & 368 & & QSLDRLMNPLIDQYL & AAV2, AAV8, AAV9 & $428-442,431-445,430-444$ & $3 F, I S$ \\
\hline$A, C, F$ & 499 & & SLDRLMNPLIDQYLY & AAV2, AAV8, AAV9 & $429-443,432-446,431-445$ & $3 F, I S$ \\
\hline $\mathrm{F}$ & 97 & & QRVSKTSADNNNSEY & AAV2 & $486-500$ & C37-2/NRV,3F,OS \\
\hline $\mathrm{F}$ & 514 & & ASSWALNGRNSLMNP & AAV9 & $506-520$ & $3 F, O S, P B$ \\
\hline $\mathrm{F}$ & 386 & & NPGIAMATHKDDEER & AAV8 & $521-535$ & VRVI, OS,PB \\
\hline $\mathrm{F}$ & 110 & & NVDIEKVMITDEEEI & AAV2 & $551-565$ & VRVII, OS \\
\hline $\mathrm{F}$ & 392 & & ARDNADYSDVMLTSE & AAV8 & $551-565$ & VRVII, OS \\
\hline$A, C, F$ & 262 & e & NLQEIVPGSVWMERD & AAV5 & $586-600$ & $3 \mathrm{~F}, \mathrm{OS}, \mathrm{PB}$ \\
\hline$A, C, F$ & 263 & e & VPGSVWMERDVYLQG & AAV5 & $591-605$ & $3 F, B$ \\
\hline $\mathrm{C}, \mathrm{F}$ & 264 & e & WMERDVYLQGPIWAK & AAV5 & $596-610$ & IS,B \\
\hline C & 401 & & GTVNSQGALPGMVWQ & AAV8 & $596-610$ & os \\
\hline C & 120 & & LPGMVWQDRDVYLQG & AAV2 & $601-615$ & IS/B \\
\hline $\mathrm{F}$ & 402 & & QGALPGMVWQNRDVY & AAV8 & $601-615$ & IS \\
\hline $\mathrm{F}$ & 277 & & QYSTGQVTVEMEWEL & AAV5 & $661-675$ & IS \\
\hline $\mathrm{C}, \mathrm{F}$ & 279 & & MEWELKKENSKRWNP & AAV5 & $671-685$ & IS \\
\hline A & 136 & & EIEWELQKENSKRWN & AAV2 & $681-695$ & IS \\
\hline$A, F$ & 418 & & VSVEIEWELQKENSK & AAV8 & $681-695$ & IS \\
\hline $\mathrm{F}$ & 419 & & EWELQKENSKRWNPE & AAV8 & $686-700$ & IS \\
\hline $\mathrm{C}, \mathrm{F}$ & 284 & & PQFVDFAPDSTGEYR & AAV5 & $696-710$ & $\mathrm{~B}$ \\
\hline
\end{tabular}


Table 1 (continued).

\begin{tabular}{lllll}
\hline Sheep & ID & Epitope Sequence & AAV Serotype & Amino acid\# (VP1 \#'s) \\
\hline F & 555 & VEFAVNTEGVYSEPR & AAV9 & Epitope/Location \\
\hline Pos
\end{tabular}

Positive hits from the peptide array were mapped on the surface of the AAV capsid to identify immunogenic regions. Five sequences that appeared frequently were designated epitopes a (MAAD), b (WHCDS), c (PADVFM), d (CLEY), e (MERD). Known AAV2 Epitopes are listed, including A20 Epitopes, A20-1, 271-HYFGYSTPWG-280; a20-2, 369-VFMVPQYGYL-378; A20-4, 566-RTTNPVATEQ-575; AAV2 A1 Epitope, 123-KRVLEPLGL-131; A69 Epitope, 171-LNFGQTGDADSV-182; AAV2 C37-B Epitopes, 493-SADNNNSEYSWT-502 AND 601-LPGMVWQDRD-610. Epitopes previously identified in Moskalenko, et al [43] are listed as MK. The location of the peptide on the AAV VP capsid structure was determined and IP-IS is Intrapentameric interface-Inner Capsid Surface, IS is Inner Capsid Surface, B is Buried in the assembled capsid, OS is outer capsid surface, PB is Partially Buried and ND is Not Determined. The inner surface residues are primarily located at interfaces, for example at monomer interfaces that make up pentermers; VRIV, VRV, VRVII, and VRVIII are surface loops that vary among serotypes as defined in Govindasamy, et al. [57] variable loops in Peptide for which there is no structural information are shaded in grey.

doi: 10.1371/journal.pone.0075142.t001

recently published longitudinal study in pediatric patients with hemophilia [48]. A few peptides listed as positive binders in Table 1 showed some reactivity to secondary antibody alone, although the signal in the control assay was still less than $10,000 \mathrm{LU}$, these peptides, peptide $44,354,355,485,486$ and 499 , represent possible false binders and were omitted from further analysis.

For AAV epitopes, conservation of the sequences recognized and their location is consistent with their reported functional roles in AAV biology. Epitope b, located on the inner surface of the capsid at the inter-pentameric interface, contains amino acids reported to be important to capsid assembly [49]. Peptides 338 and 339 common to AAV2, AAV8, and AAV9, mostly buried or located on the inside surface of the capsid also contain residues shown to be important in capsid assembly [49]. In addition, peptides 368 and 499 contain a residue $\mathrm{R} 432$ (in AAV2) involved in genome package [49]. How these regions could become exposed to trigger immunity is currently not clear, but it is possible that presentation of these epitopes could occur once proteolytic digestion of the capsid had occurred to enable peptide loading and presentation to the immune system during the course of natural infection with AAV. Thus for these buried or internally located epitopes, in the case of a natural AAV infection in sheep, antibody neutralizing mechanisms may include inhibition of capsid assembly or genome packaging. In support of a natural AAV infection in sheep, a single report in the literature does describe the isolation of what appears to be AAV-like particles [50], but no further studies were ever conducted to characterize these particles. The presence of what appears to be ovine AAV in nature thus provides a possible explanation for the presence of these cross-reactive antibodies that recognize clinically relevant serotypes of AAV in their sera. Our finding of antibodies against epitopes unique to specific serotypes of AAV suggests that multiple serotypes of ovine AAV with homology to their primate counterparts may exist in nature. Indeed, the successful isolation of multiple serotypes of porcine AAV was recently reported in abstract form [51], making this a promising area for future study. The ability of sheep to serve as a natural host for AAV makes them an attractive model for better understanding and circumventing human immunity to $\mathrm{AAV}$, since such naturally-occurring immunity may well differ from results obtained by immunizing animals with recombinant
AAV or AAV capsid-expressing vectors [47]. As a natural host for AAV, and given their closely related immunologic development to humans, it is also possible that sheep may develop memory $T$ cells, enabling them to mount cellular responses to AAV vectors, just as has been seen in human patients. Further studies will be needed to address this important possibility.

\section{Antigenic regions associated with the capsid surface}

As would be expected, the sheep serum also recognized AAV2, AAV5, AAV8, and AAV9 peptides located on the surface of their capsids. Significantly, similar capsid surface antigenic epitopes have been reported for other AAVs. For example, peptide 97 for AAV2 (Figure 4) overlaps with the epitope for AAV2 conformational antibody C37-B reported to block receptor attachment [37]. Mutations in this sequence have also been shown to affect neutralization from human sera or IVIG [52]. For AAV8, peptide 401 (Table 1; Figure 4) is located on a surface variable region, VRVIII, adjacent to the recently mapped ADK8 epitope (residue 586-591) [35]. Binding by this antibody affects infection at a post entry step. The AAV2 and AAV8 surface peptides, along with the surface peptides recognized in AAV5 (Epitope e in Table 1, Figure 4) and AAV9 (peptide 514 in Table 1, colored brown in Figure 4) are located on the protrusions which surround the icosahedral 3-fold axis of the capsid. Residues in this capsid region have been implicated in glycan receptor binding for AAV2, AAV5, and AAV9 [53-56]. Thus it is possible that inhibition of viral entry may contribute to the neutralization observed in the luciferase reporter assays. Similar to the data on the conserved peptides, these surface AAV antigenic epitopes, with sequence and structural differences, are also commonly located on the capsid and likely lead to serotype specific antibody recognition, as observed with the serum from the different sheep.

\section{Conclusions}

The naturally occurring AAV antibodies in the sheep in our study were classified as either low, moderate, or high based on the capsid ELISA. This variation is also observed in serum samples from human subjects. Thus, these sheep could serve as models for evaluating different immune suppression 


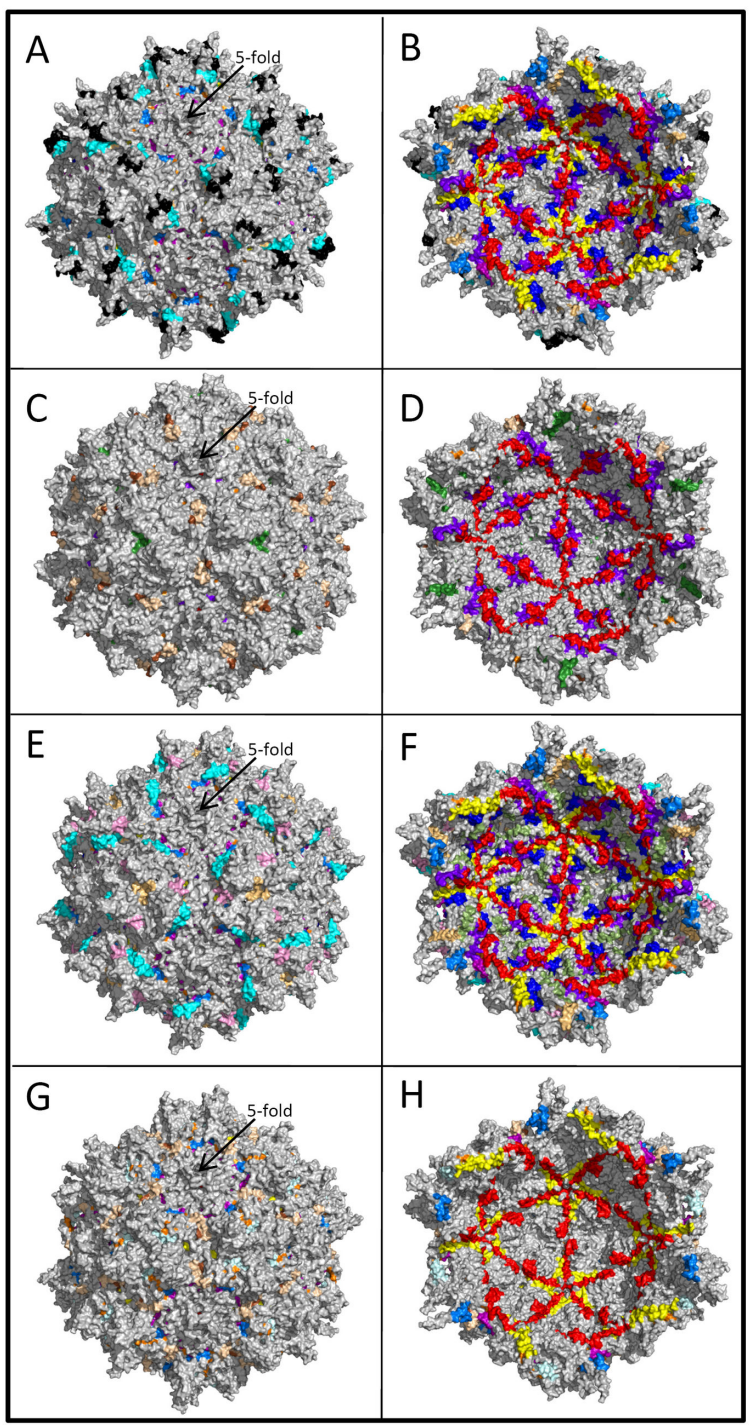

Figure 4

Figure 4. Locations of peptide epitopes on the AAV capsid. Surface density images are shown for the outside of AAV2, AAV5, AAV8, and AAV9, (A), (C), (E), and (G) respectively (left panels). Inside surface density images which are rotated $180^{\circ}$ relative the outside image and viewed approximately along the icosahedral 2-fold axis for AAV2, AAV5, AAV8 and AAV9 in (B), (D), (F), (H) respectively (right panels). Epitopes common to AAV2, AAV5, AAV8, and AAV9 are shown in red (epitope b, 221VGNSSGNWHCDSTWMGDRVI-240- Table 1), purple (epitope c, 363-PPFPADVFMIPQYGYL-378- Table 1), yellow (epitope d, 388-GRSSFYCLEYFPSQMLRTGNNFT-410 - Table 1), dark green (epitope e, 586-NLQEIVPGSVWMERDVYLQGPIWAK-610 Table 1) (RCSB PDB accession 1LP3). In A-C, the two epitopes common AAV2, AAV5, AAV8, and AAV9 are colored purple, Epitope $b$ in Table 1: 221-VGNSSGNWHCDSTWMGDRVI-240; and blue, Peptide 337 in Table 1: 273-FGYSTPWGYFDFNRF-287. In D-F, the five epitopes common AAV2, AAV8, and AAV9 are colored purple, Peptide 338 in Table 1: 278PWGYFDFNRFHCHFS-292; blue, Peptide 339 in Table 1: 283-DFNRFHCHFSPRDWQ-297; yellow, Epitope c in Table 1: 363PPFPADVFMIPQYGYL-378; red, Epitope d in Table 1: 388-GRSSFYCLEYFPSQMLRTGNN-408; and black Peptides 368 and 499 in Table 1: 428-QSLDRLMNPLIDQYLY-443. The equivalent surface accessible peptide epitopes identified for the AAV2, AAV5, AAV8, and AAV9 are shown on an AAV2 capsid surface generated with 60 copies of VP3 (accession number as given in Figure 4). The epitopes are colored differently based on the AAV serotypes: blue for AAV2 (AAV2 residues 486-QRVSKTSADNNNSEY-500, peptide 97 in Table 1), black for AAV5 (AAV5 residues 586-NLQEIVPGSVWMERD-600, peptide 262 (epitope e) in Table 1), green and dark green for two epitopes on AAV8, (green: AAV8 residues 551-ARDNADYSDVMLTSE-565, peptide 392 in Table 1; dark green: AAV8 residues 596-GTVNSQGALPGMVWQ-610, peptide 401 in Table 1), and brown for AAV9 (AAV9 residues 506ASSWALNGRNSLMNP-520, peptide 514 in Table 1).

doi: 10.1371/journal.pone.0075142.g004 
strategies prior to the administration of gene therapy vectors in efforts to optimize strategies to circumvent the immune response. The sheep also represent a useful model for evaluating re-administration of gene therapy vectors, including newly developed AAV vectors whose capsids have been intentionally modified with the goal of generating escape mutants.

To our knowledge, this is the first delineation of the epitopes within the AAV capsid that are responsible for triggering naturally-occurring, functional antibodies in these animals. The presence of these antibodies, coupled with the close parallels between human and sheep physiology, and our reestablishment of sheep with severe hemophilia A, highlight the value of sheep as a large animal model in which to study gene

\section{References}

1. Binny CJ, Nathwani AC (2012) Vector systems for prenatal gene therapy: principles of adeno-associated virus vector design and production. Methods Mol Biol 891: 109-131. PubMed: 22648770.

2. Grieger JC, Samulski RJ (2005) Adeno-associated virus as a gene therapy vector: vector development, production and clinical applications. Adv Biochem Eng/Biotechnol 99: 119-145. doi: 10.1007/10_005. PubMed: 16568890.

3. Ortolano S, Spuch C, Navarro C (2012) Present and future of adeno associated virus based gene therapy approaches. Recent Pat Endocr Metab Immune Drugs Discov 6: 47-66. doi: 10.2174/187221412799015245. PubMed: 22264214.

4. Agbandje-McKenna M, Kleinschmidt J (2011) AAV capsid structure and cell interactions. Methods Mol Biol 807: 47-92. PubMed: 22034026.

5. Mitchell AM, Nicolson SC, Warischalk JK, Samulski RJ (2010) AAV's anatomy: roadmap for optimizing vectors for translational success. Curr Gene Ther 10: 319-340. doi:10.2174/156652310793180706. PubMed: 20712583.

6. Wang J, Faust SM, Rabinowitz JE (2011) The next step in gene delivery: molecular engineering of adeno-associated virus serotypes. J Mol Cell Cardiol 50: 793-802. doi:10.1016/j.yjmcc.2010.10.017. PubMed: 21029739.

7. Sharma A, Tovey JC, Ghosh A, Mohan RR (2010) AAV serotype influences gene transfer in corneal stroma in vivo. Exp Eye Res 91: 440-448. doi:10.1016/j.exer.2010.06.020. PubMed: 20599959

8. Warrington KH Jr., Herzog RW (2006) Treatment of human disease by adeno-associated viral gene transfer. Hum Genet 119: 571-603. doi: 10.1007/s00439-006-0165-6. PubMed: 16612615

9. Gonçalves MA (2005) Adeno-associated virus: from defective virus to effective vector. Virol J 2: 43. doi:10.1186/1743-422X-2-43. PubMed: 15877812.

10. Büeler H (1999) Adeno-associated viral vectors for gene transfer and gene therapy. Biol Chem 380: 613-622. PubMed: 10430026

11. Carter PJ, Samulski RJ (2000) Adeno-associated viral vectors as gene delivery vehicles. Int J Mol Med 6: 17-27. PubMed: 10851261.

12. Nathwani AC, Tuddenham EG, Rangarajan S, Rosales C, Mclntosh J et al. (2011) Adenovirus-associated virus vector-mediated gene transfer in hemophilia B. N Engl J Med 365: 2357-2365. doi:10.1056/ NEJMoa1108046. PubMed: 22149959

13. Nathwani A, Tuddenham E, Rosales C, Mclntosh J, Riddell A et al. (2010) Early Clinical Trial Results Following Administration of a Low Dose of a Novel Self Complementary Adeno-Associated Viral Vector Encoding Human Factor IX In Two Subjects with Severe Hemophilia B. Blood 116: 248a.

14. Asokan A, Schaffer DV, Samulski RJ (2012) The AAV vector toolkit: poised at the clinical crossroads. Mol Ther 20: 699-708. doi: 10.1038/mt.2011.287. PubMed: 22273577.

15. Bartel M, Schaffer D, Buning $H$ (2011) Enhancing the Clinical Potential of AAV Vectors by Capsid Engineering to Evade Pre-Existing Immunity. Front Microbiol 2: 204. PubMed: 22065962.

16. Ito T, Yamamoto S, Hayashi T, Kodera M, Mizukami H et al. (2009) A convenient enzyme-linked immunosorbent assay for rapid screening of anti-adeno-associated virus neutralizing antibodies. Ann Clin Biochem 46: 508-510. doi:10.1258/acb.2009.009077. PubMed: 19729501.

17. Karman J, Gumlaw NK, Zhang J, Jiang JL, Cheng SH et al. (2012) Proteasome inhibition is partially effective in attenuating pre-existing immunity against recombinant adeno-associated viral vectors. PLOS therapy for hemophilia A, and other diseases, in the context of pre-existing humoral immunity to AAV. This will also aid the development of novel strategies for circumventing the immunologic barrier that has thus far thwarted the success of AAV-mediated gene transfer in the clinical arena.

\section{Author Contributions}

Conceived and designed the experiments: CDP MAM KVV VRA. Performed the experiments: CDP MAM KVV YST JF JT NT. Analyzed the data: CDP GAP MAM KVV YST VRA JF JT. Contributed reagents/materials/analysis tools: CDP GAP MAM KVV YST VRA JF JT. Wrote the manuscript: CDP GAP MAM KVV YST VRA.

ONE 7: e34684. doi:10.1371/journal.pone.0034684. PubMed: 22514654.

18. Vandenberghe LH, Wilson JM (2007) AAV as an immunogen. Curr Gene Ther 7: 325-333. doi:10.2174/156652307782151416. PubMed: 17979679.

19. Wang L, Calcedo R, Bell P, Lin J, Grant RL et al. (2011) Impact of preexisting immunity on gene transfer to nonhuman primate liver with adeno-associated virus 8 vectors. Hum Gene Ther 22: 1389-1401. doi: 10.1089/hum.2011.031. PubMed: 21476868.

20. Boutin S, Monteilhet V, Veron $P$, Leborgne C, Benveniste $O$ et al. (2010) Prevalence of serum IgG and neutralizing factors against adeno-associated virus (AAV) types 1, 2, 5, 6, 8, and 9 in the healthy population: implications for gene therapy using AAV vectors. Hum Gene Ther 21: 704-712. doi:10.1089/hum.2009.182. PubMed: 20095819.

21. Veron P, Leborgne C, Monteilhet V, Boutin S, Martin S et al. (2012) Humoral and Cellular Capsid-Specific Immune Responses to AdenoAssociated Virus Type 1 in Randomized Healthy Donors. J Immunol, 188: 6418-24. PubMed: 22593612.

22. Shin JH, Yue Y, Smith B, Duan D (2012) Humoral immunity to AAV-6, 8, and 9 in normal and dystrophic dogs. Hum Gene Ther 23: 287-294 doi:10.1089/hum.2011.125. PubMed: 22040468

23. Hurlbut GD, Ziegler RJ, Nietupski JB, Foley JW, Woodworth LA et al. (2010) Preexisting immunity and low expression in primates highlight translational challenges for liver-directed AAV8-mediated gene therapy. Mol Ther 18: 1983-1994. doi:10.1038/mt.2010.175. PubMed: 20736932.

24. Rapti K, Louis-Jeune V, Kohlbrenner E, Ishikawa K, Ladage D et al. (2012) Neutralizing antibodies against AAV serotypes 1, 2, 6, and 9 in sera of commonly used animal models. Mol Ther 20: 73-83. doi: 10.1038/mt.2011.177. PubMed: 21915102.

25. Mingozzi F, High KA (2007) Immune responses to AAV in clinical trials. Curr Gene Ther 7: 316-324. doi:10.2174/156652307782151425. PubMed: 17979678.

26. Herzog RW (2007) Immune responses to AAV capsid: are mice not humans after all? Mol Ther 15: 649-650. doi:10.1038/sj.mt.6300123. PubMed: 17372595

27. White JD, Thesier DM, Swain JB, Katz MG, Tomasulo C et al. (2011) Myocardial gene delivery using molecular cardiac surgery with recombinant adeno-associated virus vectors in vivo. Gene Ther 18: 546-552. doi:10.1038/gt.2010.168. PubMed: 21228882.

28. Mariani JA, Smolic A, Preovolos A, Byrne MJ, Power JM et al. (2011) Augmentation of left ventricular mechanics by recirculation-mediated AAV2/1-SERCA2a gene delivery in experimental heart failure. Eur J Heart Fail 13: 247-253. doi:10.1093/eurjhf/hfq234. PubMed: 21289077.

29. David AL, McIntosh J, Peebles DM, Cook T, Waddington S et al. (2011) Recombinant adeno-associated virus-mediated in utero gene transfer gives therapeutic transgene expression in the sheep. Hum Gene Ther 22: 419-426. doi:10.1089/hum.2010.007. PubMed: 20919876.

30. Porada CD, Sanada C, Long CR, Wood JA, Desai J et al. (2010) Clinical and molecular characterization of a re-established line of sheep exhibiting hemophilia A. J Thromb Haemost 8: 276-285. doi:10.1111/j. 1538-7836.2009.03697.x. PubMed: 19943872

31. Emsley P, Cowtan K (2004) Coot: model-building tools for molecular graphics. Acta Crystallogr D Biol Crystallogr 60: 2126-2132. doi: 10.1107/S0907444904019158. PubMed: 15572765. 
32. Dimattia MA, Nam HJ, Van Vliet K, Mitchell M, Bennett A et al. (2012) Structural insight into the unique properties of adeno-associated virus serotype 9. J Virol 86: 6947-6958. doi:10.1128/JVI.07232-11. PubMed: 22496238.

33. Nam HJ, Lane MD, Padron E, Gurda B, McKenna R et al. (2007) Structure of adeno-associated virus serotype 8, a gene therapy vector. J Virol 81: 12260-12271. doi:10.1128/JVI.01304-07. PubMed: 17728238.

34. Xie Q, Bu W, Bhatia S, Hare J, Somasundaram T et al. (2002) The atomic structure of adeno-associated virus (AAV-2), a vector for human gene therapy. Proc Natl Acad Sci U S A 99: 10405-10410. doi:10.1073/ pnas.162250899. PubMed: 12136130

35. Gurda BL, Raupp C, Popa-Wagner R, Naumer M, Olson NH et al. (2012) Mapping a neutralizing epitope onto the capsid of Adenoassociated virus serotype 8. J Virol, 86: 7739-51. PubMed: 22593150.

36. Lochrie MA, Tatsuno GP, Christie B, McDonnell JW, Zhou S et al. (2006) Mutations on the external surfaces of adeno-associated virus type 2 capsids that affect transduction and neutralization. J Virol 80: 821-834. doi:10.1128/JVI.80.2.821-834.2006. PubMed: 16378984

37. Wobus CE, Hügle-Dörr B, Girod A, Petersen G, Hallek M et al. (2000) Monoclonal antibodies against the adeno-associated virus type 2 (AAV-2) capsid: epitope mapping and identification of capsid domains involved in AAV-2-cell interaction and neutralization of AAV-2 infection. J Virol 74: 9281-9293. doi:10.1128/JVI.74.19.9281-9293.2000. PubMed: 10982375

38. Manno CS, Pierce GF, Arruda VR, Glader B, Ragni M et al. (2006) Successful transduction of liver in hemophilia by AAV-Factor IX and limitations imposed by the host immune response. Nat Med 12: 342-347. doi:10.1038/nm1358. PubMed: 16474400.

39. Nathwani A, [!(surname)!], Rosales C, Mclntosh J, Riddell A et al. (2010) Early Clinical Trial Results Following Administration of a Low Dose of a Novel Self Complementary Adeno-Associated Viral Vector Encoding Human Factor IX In Two Subjects with Severe Hemophilia B. Blood 116: 248a

40. Nathwani AC, Davidoff AM, Hanawa H, Hu Y, Hoffer FA et al. (2002) Sustained high-level expression of human factor IX (hFIX) after livertargeted delivery of recombinant adeno-associated virus encoding the hFIX gene in rhesus macaques. Blood 100: 1662-1669. doi:10.1182/ blood-2002-02-0589. PubMed: 12176886.

41. Scallan CD, Jiang $H$, Liu T, Patarroyo-White $S$, Sommer JM et al. (2006) Human immunoglobulin inhibits liver transduction by AAV vectors at low AAV2 neutralizing titers in SCID mice. Blood 107: 1810-1817. doi:10.1182/blood-2005-08-3229. PubMed: 16249376.

42. Mingozzi F, Anguela XM, Pavani G, Chen Y, Davidson RJ, Hiu DJ, Hinderer CJ, Faella A, Howard C, Tai A, Podsakoff GM, Ragni MV, Zhou S, Basner-Tschakarjan E, Wright JF, High KA (2012) A novel strategy to circumvent pre-existing humoral immunity to AAV. Blood 120: 2050 a.

43. Moskalenko M, Chen L, van Roey M, Donahue BA, Snyder RO et al. (2000) Epitope mapping of human anti-adeno-associated virus type 2 neutralizing antibodies: implications for gene therapy and virus structure. J Virol 74: 1761-1766. doi:10.1128/JVI.74.4.1761-1766.2000. PubMed: 10644347.

44. Zádori Z, Szelei J, Lacoste MC, Li Y, Gariépy S et al. (2001) A viral phospholipase A2 is required for parvovirus infectivity. Dev Cell 1: 291-302. doi:10.1016/S1534-5807(01)00031-4. PubMed: 11702787.
45. Popa-Wagner R, Porwal M, Kann M, Reuss M, Weimer M et al. (2012) Impact of VP1-specific protein sequence motifs on adeno-associated virus type 2 intracellular trafficking and nuclear entry. J Virol 86: 9163-9174. doi:10.1128/JVI.00282-12. PubMed: 22696661.

46. Jiménez-Clavero MA, Douglas A, Lavery T, Garcia-Ranea JA, Ley V (2000) Immune recognition of swine vesicular disease virus structural proteins: novel antigenic regions that are not exposed in the capsid. Virology 270: 76-83. doi:10.1006/viro.2000.0256. PubMed: 10772981.

47. Li H, Lin SW, Giles-Davis W, Li Y, Zhou D et al. (2009) A preclinical animal model to assess the effect of pre-existing immunity on AAVmediated gene transfer. Mol Ther 17: 1215-1224. doi:10.1038/mt. 2009.79. PubMed: 19367258

48. Li C, Narkbunnam N, Samulski RJ, Asokan A, Hu G et al. (2012) Neutralizing antibodies against adeno-associated virus examined prospectively in pediatric patients with hemophilia. Gene Ther 19: 288-294. doi:10.1038/gt.2011.90. PubMed: 21697954.

49. Wu P, Xiao W, Conlon T, Hughes J, Agbandje-McKenna M et al. (2000) Mutational analysis of the adeno-associated virus type 2 (AAV2) capsid gene and construction of AAV2 vectors with altered tropism. J Virol 74: 8635-8647. doi:10.1128/JVI.74.18.8635-8647.2000. PubMed: 10954565.

50. Clarke JK, McFerran JB, McKillop ER, Curran WL (1979) Isolation of an adeno associated virus from sheep. Brief report. Arch Virol 60: 171-176. doi:10.1007/BF01348034. PubMed: 226038.

51. Bello AJ, Chand AR, Auricchio A, Kobinger GP (2012) Characterization of Porcine Adeno-Associated Viruses Belonging to Unique Clades. Molecular Therapy 20: S46. doi:10.1038/mt.2011.197.

52. Lochrie MA, Tatsuno GP, Arbetman AE, Jones K, Pater C et al. (2006) Adeno-associated virus (AAV) capsid genes isolated from rat and mouse liver genomic DNA define two new AAV species distantly related to AAV-5. Virology 353: 68-82. doi:10.1016/j.virol.2006.05.023. PubMed: 16806384

53. Bell CL, Gurda BL, Van Vliet K, Agbandje-McKenna M, Wilson JM (2012) Identification of the galactose binding domain of the adenoassociated virus serotype 9 capsid. J Virol 86: 7326-7333. doi:10.1128/ JVI.00448-12. PubMed: 22514350.

54. Excoffon KJ, Koerber JT, Dickey DD, Murtha M, Keshavjee $\mathrm{S}$ et al. (2009) Directed evolution of adeno-associated virus to an infectious respiratory virus. Proc Natl Acad Sci U S A 106: 3865-3870. doi: 10.1073/pnas.0813365106. PubMed: 19237554.

55. Kern A, Schmidt K, Leder C, Müller OJ, Wobus CE et al. (2003) Identification of a heparin-binding motif on adeno-associated virus type 2 capsids. J Virol 77: 11072-11081. doi:10.1128/JVI. 77.20.11072-11081.2003. PubMed: 14512555.

56. Opie SR, Warrington KH Jr., Agbandje-McKenna M, Zolotukhin S, Muzyczka N (2003) Identification of amino acid residues in the capsid proteins of adeno-associated virus type 2 that contribute to heparan sulfate proteoglycan binding. J Virol 77: 6995-7006. doi:10.1128/JVI. 77.12.6995-7006.2003. PubMed: 12768018.

57. Govindasamy L, Padron E, McKenna R, Muzyczka N, Kaludov N et al. (2006) Structurally mapping the diverse phenotype of adeno-associated virus serotype 4. J Virol 80: 11556-11570. doi:10.1128/JVI.01536-06. PubMed: 16971437. 